\title{
A prevalência da incontinência urinária em mulheres praticantes de exercícios físicos de alto impacto
}

\author{
The prevalence of urinary incontinence in \\ women practicing high-impact exercises
}

\author{
Vanessa Cordeiro Mesquita' 1 \\ Mona Indianara da Costa Aragão² \\ Sabrina Amorim Correia 3 (1) \\ Amanda Silva Pereira 4 (1)
}

\section{Sylvia Christina Leite Gomes 5 Kryzia de Moraes Azevedo ${ }^{6}$ (1) Williams Silva Lima7 (1) Sávia Francisca Lopes Dias $^{8}$ (1)}

1-7Faculdade do Piauí (Teresina).Piauí, Brasil. nessa.vcm45@gmail.com, monna.aragao@gmail.com, sabrinna.amorim7@gmail.com, amandapereirasilva17@outlook.com, sylvia.clg@gmail.com, kryziaazevedo@gmail.com, willsilva16.29@gmail.com ${ }^{8}$ Autora para correspondência. Universidade Federal do Piauí. Parnaíba, Piauí, Brasil. saviadias@ufpi.edu.br

RESUMO | INTRODUÇÃO: A IU atinge cerca de 10 milhões de brasileiros de todas as idades, sendo duas vezes mais comum no sexo feminino, de acordo com a Sociedade Brasileira de Urologia (SBU). Considerada qualquer perda involuntária de urina de acordo com a ICS, podendo ser classificadas em IUU, IUE e IUM. O tratamento para a IU pode ser cirúrgico, farmacológico ou fisioterapêutico. OBJETIVO: Identificar a prevalência de incontinência urinária em mulheres praticantes de exercício físico de alto impacto, em comparação com mulheres que não praticam atividade física. MATERIAIS E MÉTODOS: O estudo foi do tipo transversal, onde se realizou um estudo de caso, sobre a perda involuntária de urina por mulheres, durante o exercício físico de impacto. As participantes foram divididas dois grupos: Grupo 1, mulheres praticantes de exercícios físicos de alto impacto e Grupo 2, mulheres não praticantes de exercícios físicos. As voluntárias realizaram o Pad Test de uma hora, padronizado pela ICS. Além disso, todas responderam o questionário Internacional Consultation on Incontinence Questionnaire - Short Form (ICIQ-SF). RESULTADOS: Houve a presença de sintomas de IUE em ambos os grupos investigados. Entretanto, foi possível comprovar que no grupo onde as mulheres praticavam exercícios de alto impacto a perda de urina foi bem maior em quantidade e em qualidade, observando-se resultados superiores a $60 \%$ nas praticantes de exercícios de alto impacto. CONCLUSÃO: As mulheres praticantes de exercícios de alto impacto apresentaram maior perda urinária comprovada pelo Pad Test e uma maior interferência desses sintomas urinários na qualidade de vida comprovada pelo ICIQ-SF.

PALAVRAS-CHAVE: Incontinência urinária. Exercícios físicos. Assoalho pélvico.
ABSTRACT | INTRODUCTION: UI affects about 10 million Brazilians of all ages, being twice as common in females, according to the Brazilian society of urology. Any involuntary loss of urine according to the ICS is considered, and can be classified into IUU, IUE and MUI. Treatment for UI can be surgical, pharmacological or physiotherapeutic. OBJECTIVE: To identify the prevalence of urinary incontinence in women, who practice high-impact physical exercise, compared to women who do not practice physical activity. MATERIALS AND METHODS: The study was a cross-sectional study, where a case study was carried out on the involuntary loss of urine by women during impact physical exercise. The participants were divided into two groups: Group 1, women practicing high-impact physical exercises and Group 2, women not practicing physical exercises. The volunteers performed the one-hour Pad test, standardized by ICS. In addition, all responded to the International Consultation on Incontinence Questionnaire - Short Form (ICIQSF). RESULTS: SUI symptoms were present in both investigated groups. However, it was possible to prove that in the group where women practiced high-impact exercises, the loss of urine was much greater in quantity and quality, with results exceeding $60 \%$ in the practitioners of high-impact exercises. CONCLUSION: Women who practice high impact exercises showed greater urinary loss as evidenced by the Pad test and a greater interference of these urinary symptoms in the quality of life proven by ICIQ-SF.

KEYWORDS: Urinary incontinence. Physical exercise. Pelvic floor. 


\section{Introdução}

A prática regular de exercícios físicos sejam esses aeróbios, de fortalecimento muscular ou de flexibilidade geralmente é indicada no processo de tratamento e na prevenção de doenças cardíacas, hipertensão, osteoporose, obesidade, diabetes, entre outras ${ }^{1}$. Além disso, tem efeitos positivos inegáveis nos aspectos emocionais, diminuindo os efeitos nocivos do estresse, alívio de tensões, melhora do humor, da ansiedade e depressão? .

Apesar de todos os benefícios já conhecidos da prática de exercícios, algumas modalidades exigem uma melhor capacidade funcional neuromuscular específica. Os exercícios de alto impacto, por exemplo, necessitam de uma maior solicitação de contenção e suporte por parte dos músculos do assoalho pélvico (MAPs) ${ }^{3}$. Disfunções nessa musculatura associadas a uma alta pressão intra-abdominal criada durante esse tipo de exercício podem resultar em episódios de perda urinária mesmo na ausência dos outros fatores de riscos${ }^{4}$.

A International Continence Society (ICS) define a incontinência urinária (IU) como toda perda involuntária de urina ${ }^{5}$. A IU é classificada como: Incontinência Urinária de Urgência (IUU) caracterizada pelo forte desejo de urinar seguida da perda de urina ${ }^{6}$; Incontinência Urinária de Esforço (IUE) que está relacionada à perda de urina devido ao aumento da pressão intra-abdominal decorrente de um esforço, quando a pressão intra-vesical é superior a pressão uretral na ausência de contração do músculo detrusor; e a Incontinência Urinária Mista (IUM) que resulta de uma combinação dos outros dois tipos ${ }^{?}$.

Na mulher, alguns fatores tendem a ser considerados predisponentes dos distúrbios urinários. A história obstétrica e ginecológica pode contribuir para o aparecimento das perdas urinárias bem como a idade, onde com o passar dos anos ocorre a diminuição das fibras colágenas, substituição de tecido muscular por tecido adiposo e consequente diminuição da força de contração dos músculos do assoalho pélvico, diminuição da capacidade de armazenamento da bexiga e baixos níveis de estrógenos na pós-menopausa-11.
Na pelve existe um conjunto de estruturas interligadas, como músculos, ligamentos, fáscias e a própria musculatura do assoalho pélvico, que são responsáveis pela sustentação da bexiga e uretra que fecham a pelve e apoiam-se nas vísceras verticalmente, toda essa junção anatômica se mantém responsável pela posição intra-abdominal e do colo vesical, portanto qualquer alteração pode interferir na continência ${ }^{12}$.

Algumas práticas esportivas e/ou laborais podem sobrecarregar, distender e interferir na habilidade da musculatura perineal em suportar esforços repetitivos, excedendo sua capacidade de sustentação e dessa forma alterando a eficácia da continência ${ }^{13}$. Nesse sentido, o exercício de alto impacto torna-se um dos fatores de risco para o desenvolvimento da incontinência urinária em mulheres fisicamente ativas atletas e não atletas ${ }^{4}$.

Entretanto, mulheres incontinentes não devem interromper a prática esportiva. Existem evidências científicas que comprovam que, o exercício fisico quando bem orientado, gera resultados positivos, reduzindo as perdas urinárias. Exercícios de baixa intensidade, por exemplo, podem ser utilizados para a prevenção ${ }^{14}$.

Esse trabalho teve por objetivo identificar a prevalência de incontinência urinária em mulheres praticantes de exercício físico de alto impacto, em comparação com mulheres sedentárias.

\section{Metodologia}

Foi realizado um estudo transversal, prospectivo que documentou a perda de urina em dois grupos de muIheres, cada grupo foi composto por 10 voluntárias e os protocolos foram realizados em uma instituição de ensino superior em Teresina, Piauí.

A coleta de dados foi realizada após a aprovação do Comitê de Ética e Pesquisa (CEP) sob o parecer $n^{\circ} 820.748$ (92698218.8.0000.5512) e da autorização pelas participantes por meio da assinatura do Termo de Consentimento Livre e Esclarecido (TCLE), conforme preconiza a resolução 466/12 do Conselho Nacional de Saúde. 
O estudo foi realizado através da captação de muIheres jovens entre 20 a 30 anos de idade, divididos em dois grupos: Grupo 1 (G1), mulheres praticantes de exercícios físicos de alto impacto (Jump, corrida, Crossfit) que praticavam os mesmos há tempo igual ou superior a 3 meses e, regularmente, no mínimo 3 dias na semana; e Grupo 2 (G2) com mulheres sedentárias há pelo menos 3 meses.

Para detectar e quantificar a perda urinária foi utilizado como método o Pad Test ou teste do absorvente. O teste baseia-se no ganho de peso do absorvente durante o período do teste em condições padronizadas e foi realizado em data e hora marcada, antes de iniciarem seus treinos. Um dia antes do teste, as participantes receberam um kit contendo uma garrafa de $500 \mathrm{ml}$ de água à temperatura ambiente, um absorvente pesado previamente em balança eletrônica de precisão com sensibilidade de $0,01 \mathrm{~g}$, e orientações sobre as atividades que seriam realizadas.

No dia do teste, as voluntárias foram orientadas a urinar duas horas antes de sua realização, utilizaram um absorvente higiênico de algodão $23 \mathrm{~cm}$ de comprimento, $7 \mathrm{~cm}$ de largura, $5 \mathrm{~mm}$ de espessura e peso médio de 5,5g. A mensuração de seu peso, ao início e ao final do teste, foi feita por balança digital Brinox capacidade de 5 kilos com a função "tara" que subtrai o peso normal do absorvente.

Inicialmente, colocou-se um absorvente com peso previamente aferido próximo ao meato uretral externo da paciente. Em seguida, a paciente ingeriu $500 \mathrm{~mL}$ de água e ficou em repouso por 15 minutos. Depois, solicitou-se que a paciente executasse determinadas ações simulando atividades da vida diária (subir e descer uma escada por 15 minutos, sentar e levantar dez vezes, tossir dez vezes, pegar objetos no chão 05 vezes, correr no mesmo lugar por um minuto e lavar as mãos em água corrente por um minuto). Após a realização das atividades propostas, o absorvente foi retirado e pesado na balança de precisão. As perdas urinárias foram avaliadas e classificadas: perdas de até $1 \mathrm{~g}$ foram consideradas insignificantes; entre 1,1 e 9,9 $\mathrm{g}$, classificadas como perdas leves; entre 10 a 49,9 g, perdas moderadas; e acima de $50 \mathrm{~g}$, perdas severas ${ }^{15}$.

As mesmas responderam ainda o questionário Internacional Consultation Incontinence
Questionnaire - Short Form (ICIQ-SF) traduzido para a língua portuguesa. O ICIQ-SF é um questionário simples, breve e auto-admissível, usado para avaliar o impacto da IU. Quanto maior o escore, maior o impacto na qualidade de vida, graduado em pontuações considerando os pontos: 0 nenhum impacto; 1 a 3 impacto leve; 4 a 6 impacto moderado; 7 a 9 grave e por último de 10 ou mais pontos muito grave.

Os dados foram analisados, tabulados com representação gráfica e comparação entre grupos pelo programa estatísticos SSP statistic, no qual foi considerado com significativo o $p<0,05$.

\section{Resultados}

A população inicial investigada foi de 30 mulheres, onde 10 foram excluídas porque estavam em período menstrual no dia do teste ou por não concordarem com o TCLE. Sendo assim, foram pesquisadas 20 voluntárias divididas em dois grupos: G1 com 10 praticantes de exercícios físicos de alto impacto e G2 com 10 mulheres sedentárias.

A idade média das mulheres do $\mathrm{G} 1$ foi de $23,2 \pm 1,32$

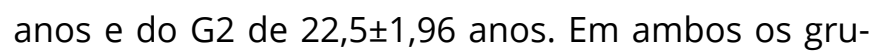
pos, $80 \%$ das mulheres eram nulíparas, $75 \%$ possuíam Ensino Superior Incompleto, 15\% possuíam Ensino Superior Completo e 10\% possuíam Ensino Médio Completo.

As atividades de alto impacto realizadas pelas voluntárias do G1 foram assim distribuídas: 20\% praticavam Crossfit, $50 \%$ corrida e $30 \%$ jump. Todas seguiram os critérios de realização do exercício com tempo de prática igual ou superior a 3 meses e frequência mínima de 3 vezes por semana.

Os sintomas urinários e o impacto da perda urinária na qualidade de vida dos grupos foram pesquisados de acordo com Internacional Consultation on Incontinence Questionnaire - Short Form (ICIQSF). O Gráfico 1 demonstra a frequência de perda de urina em relação ao grupo de acordo com a sua rotina diária e a Tabela 1 descreve a percepção da quantidade de urina perdida pelas participantes em ambos os grupos. 
De acordo com o Pad Test, $60 \%$ das praticantes de exercícios físicos apresentaram resultados positivos com os valores em gramas variando de 3,8g a 11,4g. Entre as modalidades de exercício, a que chamou mais atenção com relação a perdas de urina foi o Crossfit, na qual todas apresentaram perda de urina com a média de 8,0g. No G2, 20\% apresentou perda no Pad Test com média de 1,8g. De acordo com o ICIQ-SF é possível avaliar o impacto da IU na qualidade de vida, graduado em pontuações. Quanto mais próximo de 10, maior a interferência das perdas de urina na vida do participante. Na Tabela 2 é possível observar a diferença significativa $(p<0,05)$ no valor do Pad test e do ICIQ-SF escore entre os grupos.

Gráfico 1. Frequência de perda urinária segundo ICIQ-SF

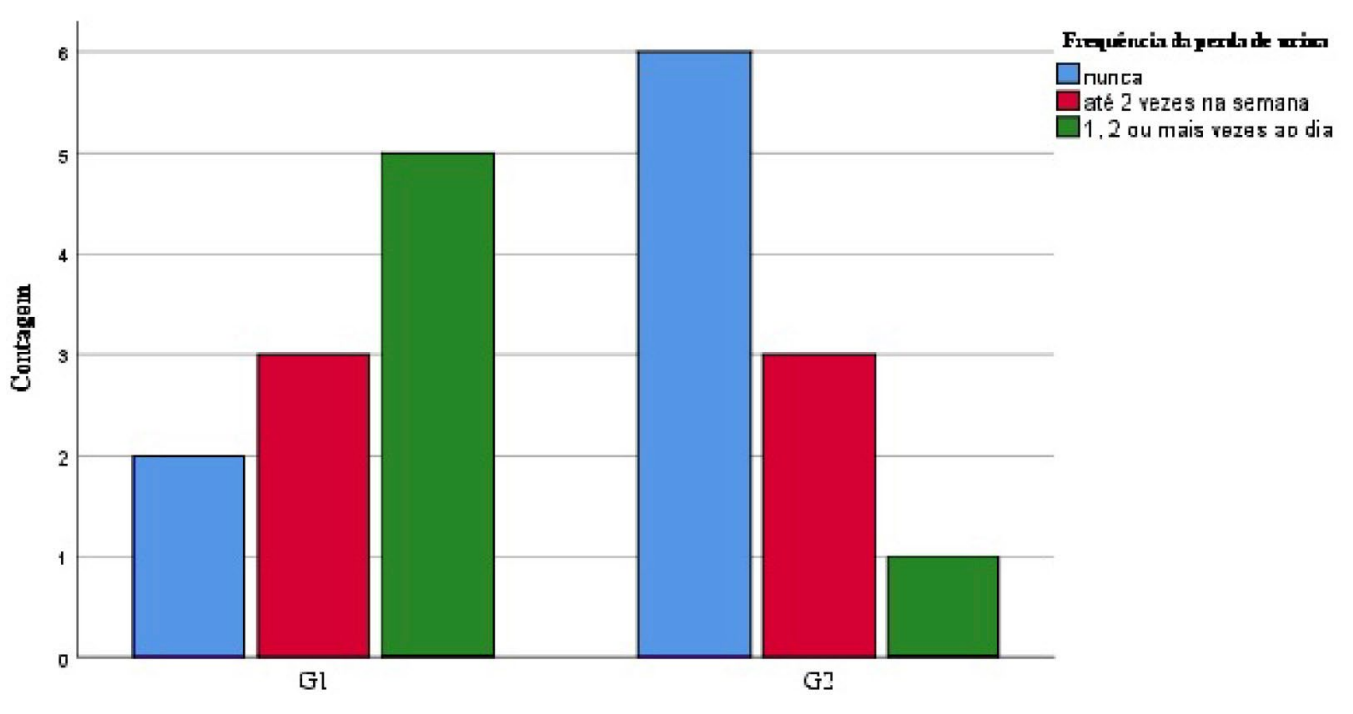

Tabela 1. Sintomas urinários dos grupos de acordo com o ICIQ-SF

\begin{tabular}{ccc}
\hline Quantidade de urina & G1 & G2 \\
\hline Pequena & $30 \%$ & $30 \%$ \\
Moderada & $40 \%$ & $10 \%$ \\
\hline Grande & $10 \%$ & - \\
\hline
\end{tabular}

Tabela 2. Dados referentes às médias do ICIQ-SF score das participantes e Pad Test

\begin{tabular}{|c|c|c|c|c|}
\hline & Grupos & Média & Desvio & $p$ \\
\hline \multirow[t]{2}{*}{ Score ICIQ-SF } & G1 & 7,60 & 1,78 & $<0,05$ \\
\hline & $\mathrm{G} 2$ & 2,20 & 0,92 & \\
\hline \multirow[t]{2}{*}{ Pad test(g) } & G1 & $7,44 \mathrm{~g}$ & 2,43 & \\
\hline & $\mathrm{G} 2$ & $1,86 \mathrm{~g}$ & 0,72 & $<0,05$ \\
\hline
\end{tabular}




\section{Discussão}

Este estudo teve por objetivo verificar a prevalência da IU em mulheres praticantes de exercícios de alto impacto em comparação com mulheres sedentárias através de dois métodos avaliativos, o Pad Test e o ICIQ-SF. Apesar de alguns pontos de imprecisão como: o número reduzido de participantes investigadas; a falta de um grupo controle composto por mulheres que realizassem outro tipo de exercício que não de impacto; e uma investigação mais aprofundada dos demais fatores envolvidos na causa da perda urinária além do exercício; foi possível comprovar que a prevalência da IU e a interferência dessas perdas urinárias na qualidade de vida nas mulheres praticantes de exercícios de alto impacto foi bem superior quando comparada ao grupo de mulheres sedentárias.

A atividade física tem sido apontada como um fator de risco para adquirir algum tipo de disfunção urinária ${ }^{16}$. Acredita-se que isso ocorra devido ao comprometimento dos mecanismos de sustentação, suspensão e contenção dos músculos do assoalho pélvico (MAP), que sofrem uma sobrecarga repetida, promovendo assim o enfraquecimento dos mesmos ${ }^{17}$. Alguns exercícios aumentam a pressão intra-abdominal, provocando uma sobrecarga para os MAP favorecendo o surgimento da perda involuntária de urina ${ }^{18}$. Os exercícios físicos de alto impacto podem ocasionar um aumento excessivo na pressão intra-abdominal, podendo sobrecarregar os órgãos pélvicos, empurrando-os para baixo, ocasionando danos aos músculos responsáveis pelo suporte desses órgãos ${ }^{19}$.

Os atletas tendem a ter uma musculatura abdominal mais forte e resistente. Ao realizar um exercício de alto impacto, por exemplo, o diafragma e os músculos abdominais contraem-se em uma maior intensidade, isso faz com que a pressão intra-abdominal se torne maior. O assoalho pélvico é quem sofre com essas repentinas cargas de forças exercidas sobre ele, pois o impacto com o solo durante o treinamento poderá revelar o enfraquecimento da musculatura pélvica, gerando uma diminuição na sua capacidade de contração, favorecendo a episódios de perdas urinárias ${ }^{20}$.
O exercício extenuante resulta em menor pressão de contração vaginal, uma vez que haveria um comprometimento do suprimento sanguíneo e debilidade de oxigênio para as fibras musculares do tipo I, responsáveis pela manutenção do tônus, indicando fadiga muscular do assoalho pélvico, além disso, existe uma redução do sinal neural após atividade muscular prolongada, reduzindo a eficiência da contração muscular favorecendo a perda da urina ${ }^{21}$.

Estudos realizados em mulheres praticantes de exercícios físicos de alto impacto envolvem diversos fatores que precisam ser analisados previamente, como o aumento da pressão intra-abdominal durante a prática da atividade física, pois apesar de algumas mulheres apresentarem a musculatura do assoalho pélvico (MAP) fortalecida, a constante prática de exercícios pode causar uma maior pressão abdominal e o enfraquecimento do assoalho pélvico, resultando na IU 22 .

Contudo, as causas da IU são multifatoriais e não podem ser limitadas exclusivamente a um único fator de risco com a prática de exercício de alta intensidade. Esses demais fatores não foram investigados nesse estudo, isso se destaca como uma potencial fonte de viés da pesquisa, entretanto os achados encontrados sugerem que o exercício pode ser sim uma dessas causas.

Outra provável etiologia da IU nas mulheres, por exemplo, está relacionada com alterações nos ligamentos cardinal, uretro-sacral e o tecido conjuntivo, que podem ser lesados e perder sua eficiência mecânica decorrente dos aumentos repetitivos da pressão abdominal, ocasionados por trabalhos manuais pesados, repetitivos e/ou tosse crônica. Mulheres jovens e nulíparas, fisiologicamente, não apresentam rupturas de ligamentos, lesão de fáscias, lesão das fibras musculares nervosas e do assoalho pélvico, a incontinência nessas mulheres pode ser oriunda de uma fraqueza genética do tecido conjuntivo, da localização abaixo do assoalho e de um número menor de fibras musculares na região ${ }^{16}$. 
Todos esses fatores justificam os resultados obtidos por esse estudo, no qual demonstrou a presença de sintomas de IUE em ambos os grupos investigados. Entretanto, foi possível comprovar que no grupo onde as mulheres praticavam exercícios de alto impacto, a perda de urina foi mais acentuada e isso interferiu diretamente na qualidade de vida. Diversos estudos já vêm mostrando uma elevada prevalência e maior risco de incontinência urinária em mulheres fisicamente ativas ${ }^{23}$ e, resultados descritos por Silva et al. ${ }^{24}$, apontam que a incontinência mais relatada dentre as atletas é a IUE, que está diretamente relacionada a lesões dos MAP, reforçando a hipótese de que as atividades de alto impacto podem ser responsáveis por causar disfunções urinárias.

A avaliação das participantes dessa pesquisa envolveu três modalidades esportivas de alto impacto diferente (Crossfit, Corrida e Jump), no total $60 \%$ das praticantes apresentaram IU comprovado pelo Pad Test. Em comparação com os resultados de Filoni E. et al. $\underline{25}$ houve similaridade, onde apresentaram resultados superiores a $54 \%$ de atletas com IU. A modalidade que se destacou, apesar da limitação amostral, foi o Crossfit, no qual todas as praticantes da modalidade apresentaram perda urinária. Em seus resultados, Yang, J. et al26 encontram para essa prática uma incidência de 50\% de IUE em mulheres jovens e menciona que por utilizar diferentes exercícios com biomecânicas específicas em cada um, o Crossfit pode ser potencialmente mais extenuante para o suporte do assoalho pélvico.

Quanto ao grupo das voluntárias sedentárias, 20\% das participantes apresentaram perda urinária significante no Pad test, o que demonstra mais uma vez que a IU envolve múltiplos fatores e estes devem ser investigados. Filoni E. et al. $\underline{25}$ encontraram resultados mais elevados, onde $40 \%$ das mulheres sedentárias perderam urina.

Na avaliação clínica, foi utilizado o Pad test, que representa uma forma objetiva de quantificação da perda urinária. Através da medida da perda de urina no absorvente, é possível classificar a incontinência urinária em leve (com perdas entre 1,1 e 9,9 g), moderada (com perdas entre 10 e 49,9 g) e severa (acima de $50 \mathrm{~g})^{26}$. O valor médio da perda urinária no grupo praticante de exercício físico foi de 7,44 g e no grupo de sedentárias foi de 1,86 g, o que confirma essa perda de urina objetivamente demonstrável27. De acordo com um estudo realizado por Dias e Rodrigues ${ }^{28}$, houve resultados semelhantes em mulheres jovens e nulíparas, concluindo que multiparidade ou envelhecimento não são únicos fatores que colaboram com a prevalência da IU.

Outro ponto investigado nesse estudo foi a interferência que as perdas urinárias provocam na qualidade de vida das mulheres. Segundo Frade et al.29, é extremamente importante avaliar o impacto e a percepção da qualidade de vida em mulheres com $\mathrm{IU}$, pois pode causar impacto negativo em amplos domínios, tais como: emocionais, físicos, mentais, relacionamento pessoal e social, bem como, alterações biológicas, afetando sobretudo a autoestima. Desse modo, podemos evidenciar os dados obtidos no estudo de Fernandes et al. ${ }^{30}$ onde descreveram que 38,4\% das mulheres investigadas relataram o quanto que a incontinência urinária pode acarretar subsídios negativos à sua qualidade de vida.

Através dos scores obtidos pelo ICIQ-SF constatou-se uma maior frequência e percepção da quantidade de urina perdida nas praticantes de exercícios físicos de alto impacto, consequentemente, tais fatores atingem de forma mais negativa na qualidade de vida dessas mulheres. Na literatura há vários estudos que corroboram esses resultados, entre eles o de Alves et al. ${ }^{31}$ e o de Carvalhais ${ }^{32}$ que mesmo avaliando modalidades esportivas diferentes, são concordantes com uma maior prevalência na frequência e quantidade de perda urinária nas atletas quando comparadas às mulheres sedentárias ou praticantes de exercícios de baixo ou médio impacto.

Segundo Ludviksdottir et al. $\frac{33}{}$, para reduzir o índice de incontinência urinária, é preciso que os treinadores se atentem ao fortalecimento e treinamento dos músculos do assoalho pélvico, pois nota-se que eles não são fortalecidos durante as sessões de treinamento geral. Caetano et al. ${ }^{34}$, também sugere que os profissionais passem a trabalhar a prevenção de IUE estabelecendo uma abordagem coadjuvante para a prevenção de incontinência urinária causada pela prática de exercícios físicos. 


\section{Conclusão}

De acordo com os dados obtidos na pesquisa, observou-se que em relação ao grupo de mulheres sedentárias, as mulheres praticantes de exercícios de alto impacto apresentaram maior perda urinária, resultado comprovado pelo Pad Test, assim como uma maior interferência negativa desses sintomas urinários na qualidade de vida das mesmas, comprovada pelo ICIQ-SF, podendo assim ocasionar prejuízos em tarefas domésticas, profissionais, influenciar na vida sexual e convívio social. Entretanto, cabe ressaltar que alguns dos sintomas característicos de incontinência urinária também foram observados no grupo de muIheres que não realizaram os exercícios. Isso sugere a existência de outros fatores que podem estar relacionados à perda urinária e, portanto, que mais estudos devem ser realizados para identificá-los.

\section{Contribuições dos autores}

Mesquita VC foi responsável por executar a coleta de dados da pesquisa, interpretar os dados e fazer a redação do manuscrito. Aragão MIC organizou o manuscrito, redigiu o resumo, contribuiu na redação da discussão e da conclusão do manuscrito, revisou o artigo. Pereira AS organizou o manuscrito, redigiu a versão em inglês do resumo, contribuiu na redação da metodologia do manuscrito, revisou o artigo. Azevedo KM e Lima WS revisaram o artigo. Correia AS e Gomes SCL revisaram a redação, interpretação dos dados e a bibliografia utilizada no manuscrito. Dias SFL concebeu o projeto, interpretou os resultados e aprovou a versão final.

\section{Conflitos de interesses}

Nenhum conflito financeiro, legal ou político envolvendo terceiros (governo, empresas e fundações privadas, etc.) foi declarado para nenhum aspecto do trabalho submetido (incluindo, mas não se limitando a subvenções e financiamentos, participação em conselho consultivo, desenho de estudo, preparação de manuscrito, análise estatística, etc.).

\section{Referências}

1. Carrol S, Dudfield M. What is therelationship between exercise and metabolic abnormalities? A review of the metabolic syndrome. Sports Med. 2004;34(6):371-418. doi: 10.2165/00007256-200434060-00004
2. Caetano AS, Tavares MCGCF, Lopes MHBM. Incontinência urinária e a prática de atividades físicas. Rev Bras Med Esporte. 2007;13(4):270-74. doi: 10.1590/\$1517-86922007000400012

3. Silva LH, Serezuella KC, Bordini A, Citadini JM. Relação da incontinência urinária de esforço com a prática de atividade física em mulheres nulíparas. Salusvita. 2005;24(2):195-218.

4. Santos ES, Caetano AS, Tavares MCGCF, Lopes MHBM. Incontinência Urinária entre estudantes de Educação Física. Rev Esc Enferm. 2009;43(2);307-12. doi: 10.1590/S0080$\underline{62342009000200008}$

5. Buckley BS, Lapitan MCM. Prevalence of urinary incontinence in men, women, and children-current evidence: findings of the Fourth International Consultation on Incontinence. Urology. 2010;76(2); 265-70. doi: 10.1016/j.urology.2009.11.078

6. Haylen BT, Ridder D, Freeman RM, Wift SE, Berghmans B, Lee J et al. An International Urogynecological Association (IUGA)/ International Continence Society (ICS) Joint Report on the Terminology for Female Pelvic Floor Dysfunction. Int Braz J Urol. 2010;21(1):5-26. doi: 10.1590/S1677-55382010000100032

7. Dedicação AC, Haddad M, Saldanha MES, Driusso P. Comparison of quality of life for different types of female urinary incontinence. Rev Bras Fisioter. 2009;13(2):116-22. doi: 10.1590/ $\underline{\text { S1413-35552009005000014 }}$

8. Aslan E, Beji NK, Erkan HA, Yalcin O, Gungor F. Urinary incontinence and quality life of the elderly residing in residential homes in Turkey. Arch Gerontol Geriatr. 2009;49(2):304-310. doi: 10.1016/j.archger.2008.10.009

9. Dellu MC, Zacaro PMD, Schmitt ACB. Prevalência de sintomas urinários e fatores obstétricos associados em mulheres adultas. Rev Bras Fisioter. 2008;12(6):482-487. doi: 10.1590/S141335552008005000005

10. Figueiredo EM, Lara JO, Cruz MC, Quintão DMG, Monteiro MVC. Perfil sociodemográfico e clínico de usuárias de serviço de fisioterapia uroginecológica da rede pública. Rev Bras Fisioter. 2008;12(2):136-142. doi: 10.1590/\$1413-35552008000200010

11. Chen Y, Chen GD, Hu SW, Lin TL, Lin LY. Is the occurrence of storage and voiding dysfunction affected by menopausal transition or associated with the normal aging process? Menopause. 2003;10(3):203-8. doi: 10.1097/00042192-200310030$\underline{00005}$

12. Botelho F, Silva C, Cruz F. Incontinência Urinaria Feminina de urologia do hospital de São João. Acha Urológica. 2017;24(1):7982.

13. Matheus LM, Mazzari CF, Mesquita RA, Oliveira J. Influência dos exercícios perineais e dos cones vaginais associado à correção postural no tratamento da incontinência urinaria feminina. Rev Bras Fisioter. 2006;10(4):387-92. doi: 10.1590/ S1413-35552006000400005 
14. Martines GA, Tamanini JTN. Relação entre atividade física e incontinência urinaria: informações relevantes ao educador físico. Saúde e Pesquisa. 2015;8(1):149-155. doi: 10.17765/2176-9206.2015v8n1p149-155

15. Albuquerque MT, Micussi BC, Soares EMM, Lemos TMAM, Brito TNS, Silva JB et al . Correlação entre as queixas de incontinência urinária de esforço e o pad test de uma hora em mulheres na pós-menopausa. Rev Bras Ginecol Obstet. 2011;33(2):70-4. doi: 10.1590/S0100-72032011000200003

16. Caetano AS, Tavares MCGCF, Lopes MHBM. Incontinência Urinária e a Prática de Atividade Física. Rev Bras Med Esporte. 2007;13(4):270-274.

17. Virtuosa JF, Menezes EC, Mazo GZ. Fatores de risco para Incontinência Urinária em mulheres idosas praticantes de exercícios físicos. Rev Bras Ginecol Obstet. 2015;37(2):82-86. doi: 10.1590/SO100-720320140005040

18. Korelo RIG, Kosiba CR, Grecco L, Matos RA. Influência do fortalecimento abdominal na função perineal, associado ou não à orientação de contração do assoalho pélvico, em nulíparas. Fisioter Mov. 2011;24(1):75-85. doi: 10.1590/S0103$\underline{51502011000100009}$

19. Goldstick O, Constantini N. Urinary incontinence in physically active women and female athetes. $\mathrm{Br} J$ Sports Med. 2014;48(4):296-8. doi: 10.1136/bjsports-2012-091880

20. Santos AM, Silveira HRM, Lima TVS, Pontes IEA. Ocorrência de incontinência urinária em praticantes de esportes de alto impacto: uma revisão integrativa da literatura. Temas em Saúde. 2020;20(2):286-314. doi: 10.29327/213319.20.2-16

21. Almeida PP, Machado LRG. A prevalência de incontinência urinária em mulheres praticantes de jump. Fisioter Mov. 2012;25(1):55-65. doi: 10.29327/213319.20.2-16

22. Andrade NVS, Manso VMC, Antunes MB. Incontinencia Urinaria de esforço em mulheres praticantes de atividades físicas. Rev Ensaios e ciências biológicas, agrárias e da saúde. 2011;15(3):41-53.

23. Casey EK, Temme K. Pelvic floor muscle function and urinary incontinence in the female athlete. Phys Sportsmed. 2017;45(4):399-407. doi: 10.1080/00913847.2017.1372677

24. Silva RM, Santos MP, Araújo MP, Sartori MG, Resende APM. A prática esportiva e o assoalho pélvico feminino: uma revisão da literatura. Arq Cien Esp. 2019;7(1):2-7. doi: 10.17648/aces. v7n1.3502
25. Filoni E, Capato FCA, Fitz FF, Fernandes AO, Sens YAS. Comparação de esportes de Alto impacto e Baixo impacto em relação á Incontinência Urinária. Rev Vit et Sanitas. 2015;9(1):7374.

26. Yang J, Cheng JW, Wagner H, Lohman E, Yang SH, Krishingner GA et al. The effect of high impact crossfit exercises on stress urinary incontinence in physically active women. Neurourol Urodyn. 2019;38(2):749-756. doi: 10.1002/nau.23912

27. Soroka D, Drutz HP, Glazener CMA, Hay-Smith EJC, Ross S. Perineal pad test in evaluating outcome of treatments for female incontinence: a sistematic review. Int Urolgynecol J Pelvic Floor Dysfunct. 2002;13(3):165-175. doi: 10.1007/s192-002-8348-z

28. Dias SFL, Rodrigues AMS. A prevalência da incontinência urinaria em mulheres nulíparas. J Health Sci Inst. 2016;34(1):4952.

29. Frade AB, Frade CL, Leite TG, Domenich TR, Auge APF. Modified pubovaginal sling technique in the surgical management of female stress urinary incontinence. Rev Col Bras Cir. 2015;42(6):377-381. doi: 10.1590/0100-69912015006005

30. Fernandes S, Coutinho EC, Duarte JC, Nelas PAB, Chaves $\mathrm{CMCB}$, Amaral O. Quality of life in women with Urinary Incontinence. Rev. Enf. Ref. 2015; 4(5):93-99. doi: 10.12707/ $\underline{\text { RIV14042 }}$

31. Alves JO, Luz ST, Brandão S, Luz CM, Jorge RN, Roza T. Urinary incontinence in physically active younh women: Prevalence and related factors. Int J Sports Med. 2017;38(12):937-941. doi: 10.1055/s-0043-115736

32. Carvalhais A, Jorge RN, BØ K. Performing high-level sport is strongly associated with urinary incontinence in elite athletes: a comparative study of 372 elite female athletes and 372 controls. Br J Sports Med. 2018;52(24):1586-1590. doi: 10.1136/ bjsports-2017-097587

33. Ludviksdottir I, Hardardottir H, Sigurdardottir T, Ulfarsson GF. Comparison of pelvic floor muscle strength in competition-level athletes and untrained women. Laeknabladid. 2018; 104(3):133138. doi: $10.17992 / / \mathrm{bl} .2018 .03 .177$

34. Caetano AS, Suzuki FS, Lopes MHBM. Urinary Incontinence and Exercise: Kinesiological Description of an Intervention Proppsal. Rev Bras Med Esporte. 2019;25(5):409-412. doi: $\underline{10.1590 / 1517-869220192505213379}$ 